in this case could be found. The fact, also, that rhinoscleroma has been observed in this country only in persons who have come from districts in which the disease is endemic renders it highly improbable that my patient's ailment is of this nature. Lastly, the absence of the specific bacillus and of Mikulicz's cells almost conclusively disproves rhinoscleroma.

2. Tertiary syphilis, owing to the various aspects it may assume in the throat, naturally suggests itself. Inquiry as to the patient's previous health yields no indication of his ever having contracted venereal disease; besides, this morbid process differs from tertiary syphilis in its perfect symmetry, in the absence of any cendency to ulceration, and in its being uninfuenced by iodide of potassium.

3. Hereditary syphilis. - Recently I have had under treatment a boy, aged seven years, the subject of. late hereditary syphilis. In addition to laryngeal manifestations his palate and uvula presented a smooth uniform thickening which, although less marked, resembled that of the case under consideration. The greater part of the uvula was removed and Dr. Ferguson found, on examining it microscopically, that the enlargement was due to a diffuse fibroid hyperplasia which in its main features closely resembled the histological appearances presented in sections from the case the subject of this paper.

In consequence of the similarity of the histological appearances in the two uvalas it has been necessary to consider-(1) whether the man is the subject of late hereditary syphilis, and (2) whether the sclerotic hyperplasia in the child is independent of syphilis.

I have failed to discover any fact in support of the first question. The patient's parents spent their married life in the Highlands and had a family of 12 children, all of whom reached adult life, the physique of every member being above the average. The patient is the second youngest child and presents none of the stigmata of the diathesis. Owing to the want of corroborative evidence in the perronal and family history of the patient, and because of the general dissimilarity of his pharyngeal manifestations to those observed in hereditary syphilis, I believe that this disease may be left out of account as an etiological factor.

The second question, as to whether the involvement of the pharynx in the boy is independent of syphilis, must be answered in the negative. That he suffered from hereditary syphilis when an infant is beyond doubt. It is also evident that the affection of his pharynx is of the same nature as that of his larynx and that the latter resembles the diffuse hyperplastic infiltration of the larynx which has been described in a few cases of hereditary syphilis. On these grounds the hyperplasia of the boy's uvala may be attributed to the diathesis.

While I have been unable to find a disease of which the sclerotic hyperplasia in the man is a manifestion, I would point out that an analogous condition probably exists in subglottic hypertrophic laryngitis. This affection is usually characterised by the presence of pinkish, smooth, firm, symmetrical folds beneath the vocal cords, which develop slowly, apparently in consequence of recurrent inflammatory attacks, during which they become more or less swollen. If we now turn to the case under discussion we find folds of thickened tissue in the pharynx presenting characters similar to those just mentioned; we get a clinical history of his having been subject to sore-throat for years, and of permanent symptoms due to hypertrophic changes having set in only at a comparatively recent date; examination during an inflammatory attack revealed marked swelling of part of the affected region; and, lastly, Dr. Ferguson informs me that $A$. Sokolowski's $\mathbf{s}^{1}$ account-which is probably the most detailed and thorough-of the histology of subglottic hypertrophic laryngitis might stand for that of the removed portion of the enlarged uvula, the sole difference being that in our case the deeper layer of the epithelium is not thrown into papillæ, but presents practically an even continuous surface, the columnar character of the deepest cells of the rete Malpighii, however, being maintained.

Considerable discussion has taken place as to the nature of subglottic hypertrophic laryngitis. In most text-books the causes mentioned are tuberculosis, syphilis, and rhinoscleroma, the last being probably the commonest. Of 100 cases of scleroma recently reported by $A$. Baurowicz ${ }^{2} 34$

Ein Beitrag zur Pathologie und Therapie der chronischen hypertrophischen Kehlkopfentzindung, Arehiv fïr Laryngologie, Band ii., 8. 68 ; also Band iv., S. 239.

2 Das Sklerom auf Grund der Beobachtung von 100 Fällen, Archiv fur Laryngologie, Band x., S. 396; Zur Aetiologie der sogenannten were affected with subglottic hypertrophic laryngitis. IIf the frequency of subglottic hypertrophic laryngitis in scleroma be coupled with its great rarity apart from this disease it can be understood how certain observers who live where rhinoscleroma is prevalent-e.g., P. Pieniazek ${ }^{3}$ and Baurowicz of Cracow-and who consequently see subglottic hypertrophic laryngitis comparatively often, maintain that it is alvays a manifestation of scleroma. On the other hand, Sokolowski of Warsaw, who has also had an extensive experience of rhinoscleroma, denies this, and proves incontestably, as it seems to me, that subglottic hypertrophic laryngitis may develop independently of scleroma, tuberculosis, and syphilis; he thinks that the morbid process is of a specific nature, the etiological factor being as yet unknown, but he suggests that hereditary syphilis may play a part. $\Delta$. Kuttner ${ }^{4}$ reports a case of subglottic hypertrophic laryngitis in which there were no grounds for suspecting the presence of any of the infective diseases mentioned; he maintains, therefore, that this laryngeal condition may appear as an affection suigeneris which he proposes to term the genuine form of chorditis vocalis inferior hypertrophica. F. $H$. Bosworth ${ }^{5}$ also states that there can be no question of a simple idiopathic inflammatory process in the subglottic region giving rise to marked hypertrophy, but he thinks that in most instances a diathetic condition is present. There are thus good reasons for according to subglottic hypertrophic laryngitis independent rank amongst the diseases of the larynx.

The facts brought forward in this paper, if correctly interpreted, prove (1) that the pharynx and naso-phargnx may be the seat of a sclerotic hyperplasia unconnected with syphilis, rhinoscleroma, or other known infective disease : (2) that a similar morbid process may manifest itself beneath the vocal cords as subglottic hypertrophic laryngitis; and (3) that in the hyperplastic variety of hereditary syphilis the histological appearances closely resemble those of the above described sclerotic hyperplasia.

Glasgow.

\section{ON THE EXISTENCE OF IMMUNITY AFTER ENTERIC FEVER.}

BY BURTON A. NIOOL, M.R.O.S. ENG., L.R.C.P. LOND. GIVIL SURGEON ATTACHED TO THE ROYAL ARMT MRDICAI CORPS, SOUTH AFRICAN FTELD FORCE; IATE HOUSE PHYSICIAN, CHARING-CROSS HOSPITAL.

THERE seems to be a very general impression that an attack of enteric fever confers some degree of immunity upon the patient against further attacks of the same disease. The number of patients whom I found suffering from enteric fever in South Africa who had previously contracted the disease in India or elsewhere has made me feel that this conclusion is not based upon very good evidence.

A nursing sister of my acquaintance had two attacks of enteric fever in England during her nursing career, and she contracted the disease for the third time whilst doing duty with the forces in South Africa. An officer had suffered from enteric fever in India, and he also contracted malaria in that country. During the campaign he again became a victim and his illness was followed by thre distinct relapses. These relapses did not occur in the usual way during the week or so after the tem. perature became normal, but came on when the patient was on an improved diet and beginning to get abouti.e., from three to five weeks after the temperature had fallen. This was, of course, altogether an unusual case and may have been complicated by his malarial diathesis. The following two cases are interesting as showing that the supposed immunity produced by very severe attacks of enteric fever was not sufficient to protect the patients for more than six months from a recurrence of their illnesses.

CASE 1.-A civil surgeon, aged 30 years, previonsly in good health, was admitted to No. 7 General Hospital aq Estcourt in May, 1900, with symptoms of enteric fever. The attack was a most virulent one, the patient suffering from

Chorditis vocalis inferior hypertrophica, Archiv für Laryngologie, Band vii., S. 349 .

Heymann's Handbuch der Laryngologie, Band i., S. 1305

4 Chorditis vocalis inferior hypertrophica, Archiv für Laryngo ogie, Band v., S. 275 .

5 A Treatise on Diseases of the Nose and Throat, vol. ii., p. 529. 
very active delirium during the whole time that the temperature was raised. He had eight distinct hæmorrhages within a few days and the collapse produced by loss of blood was so extreme that he was not expected to recover. The temperature for about a week was between $103^{\circ}$ and $105^{\circ} \mathrm{F}$. He rallied, the temperature fell to normal on the eighteenth day, and from that time he made a good convalescence. He was sent home to Fngland and after spending a month at home he returned to duty. His blood was examined during the illness for the Widal reaction; the clumping and loss of motility of the bacilli were instantaneous. In November, 1900, whilst doing duty at No. 14 General Hospital at Newcastle, this patient again went to bed with every symptom of enteric fever. He had headache, diarrhcea, and during the first week considerable delirium. The temperature ranged for some days between $103^{\circ}$ and $104.5^{\circ}$ and did not become for 30 days. A very typical roseolar eruption appeared over the whole front of the chest and the abdomen, the back, and the extremities, thus covering every part of the body with the exception of the head and neck. Althongh the illness was rather a long one it was not regarded as being unusually severe in type and the patient again made an uninterrupted recovery. The Widal reaction was again tried, this time about 10 weeks after the temperature fell. The result was a positive reaction, clumping and loss of motility taking place in about 20 minutes with the usual dilution.

CASE 2.-A private in the 2nd Middlesex Regiment, aged 24 years, was sent from a field hospital to No. 7 General Hospital at Estcourt in April, 1900. On admission he had a temperature ranging from $102^{\circ}$ to $1035^{\circ} \mathrm{F}$., was typhoidal in appearance, and had a slight abdominal rash, abdominal fulness, and diarrhce. The type of enteric fever was considered to be quite of average severity. His temperature fell to normal on the twenty-second day, but after remaining normal for four days it again rose, finally falling on the thirty-fourth day of the illness. Beyond this relapse there were no complications. He made a good recovery and was discharged to the convalescent depôt. This patient ought to have gone to England, but some mistake was made, and after remaining at the convalescent depôt for a month or two he was with others sent back to his regiment at the front. In September, 1900, he again had symptoms of enteric fever and was sent into hospital at Newcastle. On this occasion the attack was quite a mild one, and there was no relapse or any sort of complication. The temperature never went beyond $103^{\circ}$ and fell to normal on the nineteenth day. He made an uninterrapted convalescence and on this occasion was sent to England. The Widal reaction was not attempted with this patient in either illness.

In these two cases the attack may have been due to an auto.infection. It is quite conceivable that the bacillus may remain within the body quiescent for many months and only become active at some time when, owing to fatigue, exposure, or other causes the bodily resistance to disease is temporarily lowered. If the natural immunity acquired by a very severe attack of the disease is of any value, however, the patient ought to be protected against the disease whether the cause comes from within or from without. One would also expect to find the degree of immunity varying directly with the intensity of the primary attack.

It is acknowledged by most observers that immunity acquired in this manner, if it exists, is much less pronounced and of more doubtful duration than that produced by other febrile disorders, such as small-pox, scarlet fever, \&c. The question of its existence is of considerable importance at the present time as the possibility of procuring an artificial immunity by the present method of inoculation is at least doubtful, if we are not satisfied that an attack of the disease itself protects its victim against further onsets.

The clinical evidence upon the point is necessarily weak. Under ordinary circumstances the cause of the disease is only very occasionally present, and when it is present the predisposing circumstances favouring its development may be entirely absent. The large majority of the population at home, therefore, escape the disease altogether, and it is consequently not surprising if very few of those who have had the disease once contract it a second time. The present campaign will not throw any light upon the problem, as no systematic inquiry has been made as to whether the patient has or has not suffered from previous attacks of enteric fever. Most medical officers, however, have gained the impression that second attacks of enteric fever are fairly frequent, and even third attacks are not unknown. The peculiar frequency of relapses make the consideration of possible immunity much more complex. If the disease produces in its subject changes in the blood-serum antagonistic to the bacilli or their products one would not expect to find relapse so common a phenomenon in the clinical picture.

The experimental or laboratory evidence of an immunity existing consists mainly of the fact that the blood-serum of a patient who is suffering from enteric fever or who has had the disease recently has the power, even when considerably diluted, of interfering with the motility of Kberth's bacillus, and producing that characteristic clumping or agglutination of the bacilli which is known as the Widal reaction. This reaction is a very variable one. The same result has been obtained with the blood-serum of patients suffering from influenza, pneumonia, acute miliary tuberculosis, \&c., and the suggestion that these patients at some time in their previous history have suffered from an undetected or abortive attack of enteric fever does not seem altogether to meet these cases. The reaction is also obtained with the serum from patients suffering from bacillus coli infection. The serum of the normal subject if not sufficiently diluted is also not without its effect upon Eberth's bacillus and there is no constant ratio at any time between the intensity of the disease and the intensity of the reaction. Various observers have recorded cases presenting clinically all the appearances of enteric fever but in which the blood-serum gave a negative reaction.

The Widal test is doubtless of great value from a diagnostic point of view, but the very striking fact that serum from patients who have been inoculated produces loss of motility and clumping of bacilli is certainly no guarantee that an artificial immunity has been produced in these cases. A very large number of the cases of enteric fever occurred in young soldiers soon after their arrival in South Africa and amongst these were many who had been inoculated on board ship during the voyage out and in whom the protection being so recent ought to have been particularly good. The very first case admitted into No. 7 General Hospital at Estcourt was one of our own orderlies suffering from enteric fever; he had been inoculated some three weeks previously during the voyage out.

It seems to me that at present there is no proof existing of the presence of an immunity after an attack of enteric fever. Immunity varies so much in different diseases and in different individuals : erysipelas leaves behind it apparently a predisposition to further attacks; diphtheria and pneumonia as far as we know give no protection; whilst an attack of one of the exanthemata usually protects its subjects very effectually. What place enteric fever is to take in this respect has still to be determined. My recent clinical experiences have led me to doubt the existence of any immunity in many cases. A large number of men in this campaign have, how ever, quite escaped the onslaughts of enteric fever and it is reasonable to think that all must frequently have been exposed to the risks of infection. If we can discover the reason for their resistance to the disease we may then hope to discover some method of general protection. Statistics may show that inoculation is a step in the right direction, but meanwhile we should not forget that the teachings of sanitary science to-day point out clearly many paths along which efforts at prevention can be successfully made.

THE PATHOLOGY AND TREATMENT OF RHEUMATOID ARTHRITIS.

\author{
By P. W. LATHAM, M.D. OANTAB,
}

FELLOW AND LATE SENIOR GENGOR OF THE ROYAL COLLEGE OF PHYSICIANS OF LONDON; CONSULTING PHYSICIAN TO ADDENBROOKE'S HOSPITAI, CAMBRIDGK.

THE obscurity which envelopes the pathology of rheu. matoid arthritis and the doubts which are entertained as to the effects resulting from the administration of drugs induce me to invite further attention both to the pathology and the treatment of this disease.

Notwithstanding the fact that the dystrophic or neural theory is said to be unsupported hitherto by any positive evidence of morbid change in the nerve cells of the spinal cord or in the nerves which supply the joints, I can find no evidence in post-mortem records that either the spinal cord or the sympathetic ganglia have been so carefully examined by modern methods and 